\title{
Quantification of Network Perfusion in ASL Cerebral Blood Flow Data with Seed Based and ICA Approaches
}

\author{
Kay Jann • Ariane Orosz • Thomas Dierks • \\ Danny J. J. Wang $\cdot$ Roland Wiest $\cdot$ Andrea Federspiel
}

Received: 16 November 2012/Accepted: 7 March 2013/Published online: 19 March 2013

(C) Springer Science+Business Media New York 2013

\begin{abstract}
Independent component analysis (ICA) or seed based approaches (SBA) in functional magnetic resonance imaging blood oxygenation level dependent (BOLD) data became widely applied tools to identify functionally connected, large scale brain networks. Differences between task conditions as well as specific alterations of the networks in patients as compared to healthy controls were reported. However, BOLD lacks the possibility of quantifying absolute network metabolic activity, which is of particular interest in the case of pathological alterations. In contrast, arterial spin labeling (ASL) techniques allow quantifying absolute cerebral blood flow (CBF) in rest and in task-related conditions. In this study, we explored the ability of identifying networks in ASL data using ICA and to quantify network activity in terms of absolute CBF values. Moreover, we compared the results to SBA and performed a test-retest analysis. Twelve healthy young subjects performed a fingertapping block-design experiment. During the task pseudo-continuous ASL was measured. After CBF quantification the individual datasets were concatenated and subjected to the ICA algorithm. ICA proved capable to identify the somato-motor and the default mode network. Moreover, absolute network CBF
\end{abstract}

K. Jann $(\varangle) \cdot$ A. Orosz · T. Dierks · A. Federspiel Department of Psychiatric Neurophysiology, University Hospital of Psychiatry, University of Bern, Bolligenstrasse 111, 3000 Bern 60, Switzerland

e-mail: jann@puk.unibe.ch

K. Jann · D. J. J. Wang

Department of Neurology, Ahmanson-Lovelace Brain Mapping Center, UCLA, Los Angeles, CA, USA

R. Wiest

Department of Diagnostic and Interventional Neuroradiology, Inselspital and University of Bern, Bern, Switzerland within the separate networks during either condition could be quantified. We could demonstrate that using ICA and SBA functional connectivity analysis is feasible and robust in ASL-CBF data. CBF functional connectivity is a novel approach that opens a new strategy to evaluate differences of network activity in terms of absolute network CBF and thus allows quantifying inter-individual differences in the resting state and task-related activations and deactivations.

Keywords Functional connectivity - Independent component analysis - Seed based analysis - Arterial spin labeling · Network quantification - Cerebral blood flow

\section{Introduction}

The functions of the brain can be thought of as complex networks optimized both for segregated and distributed information processing. The identification and investigation of functionally connected networks (FCN) exhibiting synchronized low frequency fluctuations using seed based analyses (SBA) or independent component analysis (ICA) has found its application in a wide range of basic and clinical research questions (Broyd et al. 2009; Cole et al. 2010; Greicius 2008; Jann et al. 2010b). SBA is typically hypothesis driven as it requires the a priori specification of a seed region (Biswal et al. 1995; Joel et al. 2011). Computation of the functional connectivity is then based on general linear models (GLMs) with the timecourse of the seed serving as a regressor. In contrast, decomposition of the measured signal into independent components (ICs) is a completely data driven approach and results in a spatial map and the associated temporal dynamics for each IC. Thereby, specific ICs coding for functionally meaningful networks are separated from the signal part representing 
artifacts, noise and other non-physiological drifts. FCNs representing motor, auditory and visual systems as well as higher cognitive functions like attention and working memory have been repeatedly described (Beckmann et al. 2005; Cole et al. 2010; Damoiseaux et al. 2006; Jann et al. 2010b). During task execution two classes of FCNs were described: Task positive networks that show increased activity during periods of task execution and task negative networks that show reversed temporal dynamics and are more active during periods of rest (Fox et al. 2005; Raichle and Snyder 2007). The major and most studied task negative FCN is the so-called default mode network (DMN). Initially, the DMN was identified in PET data as areas that exhibited consistently less metabolic activity across several studies and different tasks (Raichle et al. 2001). However, due to several reasons, functional network analysis is nowadays almost exclusively performed in fMRI blood oxygenation level dependent (BOLD). The wide availability of MR scanners, the non-invasiveness and repeatability of the measurements (no radioactive tracers like in PET), the lower cost of MRI as compared to PET investigations and the higher temporal resolution of the BOLD signal that allows investigating also temporal characteristics of the networks have favoured this shift from PET to MR. Besides these evident advantages offered by BOLD there is one major disadvantage compared to PET. Since the BOLD signal relies on relative changes of the ratio of blood oxygenation only relative measures of the network's activity can be estimated, whereas PET provides the possibility to quantify absolute measures for the metabolic network activity. Moreover, the BOLD signal is modulated by other physiological factors such as cerebral blood flow (CBF), cerebral blood volume (CBV) and cerebral metabolic rate of oxygen (CMRO2) and thus is a rather indirect measure of neural activity. Therefore, the assessment of a more direct measure of neural activity in this model of the hemodynamic response to brain activation (Buxton et al. 2004; Duong et al. 2001) that also allows quantifying absolute values in addition to temporal fluctuations would be of high interest. Such measures of absolute network activity would especially be crucial as to the understanding of pathophysiological alterations and neurological disorders (Alzheimer's disease, Parkinson's disease, dementia, schizophrenia, depression) but could also help to better understand the relation between task positive and task negative FCNs.

Arterial spin labeling (ASL) is a non-invasive functional MRI method that uses blood water as endogenous tracer by magnetic labeling of the water proton spins (Detre et al. 1992; Williams et al. 1992). Moreover, ASL combines the advantages of both BOLD MR and PET. ASL has been shown to yield replicable absolute quantified values of cerebral perfusion comparable to the results obtained with $\mathrm{H}_{2}^{15} \mathrm{O}$-PET in patient populations and in healthy controls
(Bokkers et al. 2008, 2010; Ye et al. 2000). It allows for repeated acquisitions since no exposure to radioactivity is required and it provides a temporal resolution in the range of a few seconds. However, the available ASL sequences generally have a relatively low signal-to-noise ratio (SNR) although newer sequences are being developed to increase the sensitivity. The quantification of ASL requires subtraction of the label image from the control image (Luh et al. 1999). This subtraction not only halves the number of images recorded, which poses a limitation to the amount of data that can be acquired in each session, it also halves the temporal resolution with regard to the TR of the sequence. Nevertheless, it is still within the range of seconds and thus sufficient to capture the dynamics of FCNs (low frequency fluctuations $0.01-0.08 \mathrm{~Hz}$ ) (Chuang et al. 2008). The limited length of the quantified dataset (typically not more than 100 quantified images) together with the low SNR makes it challenging to reliably decompose perfusion image series into FCNs in single subject analyses. Hence, in most applications of ASL, mean CBF maps averaged across the duration of acquisition were computed and the temporal dynamics were not considered. A handful of previous studies however analyzed the temporal signal and the functional connectivity of ASL datasets (Biswal et al. 1997; Chuang et al. 2008; Wu et al. 2009; Zou et al. 2009). These studies demonstrated the feasibility to detect functionally connected networks, such as the motor network (Biswal et al. 1997; Chuang et al. 2008) and the DMN (Wu et al. 2009; Zou et al. 2009). But noteworthy, all these studies applied the temporal correlation approach using SBA to identify the FCNs. To date, the feasibility to identify FCNs within perfusion time series using ICA has been described in a couple of works (Liang et al. 2012; Dai et al. 2012) but a comparison to the SBA or a test-retest analysis is still missing.

The temporal concatenation of datasets as proposed by Calhoun et al. (2004) which has become a standard procedure in BOLD fMRI group ICA, might overcome the problems of SNR and limited number of observations in individual datasets and thus might render it possible to estimate FCNs with ICA in perfusion time series data. Therefore, the aim of this study was to identify task related networks in ASL data using a concatenated group ICA and to contrast them with the SBA results. Performance of a task helps to increase further the SNR within the dataset as compared to pure resting state recordings. To this end, subjects performed a simple motor task following a block design. We further computed the subject specific maps (SMs) based on the group ICA results and the SBA to test the robustness of the approaches. Moreover, we assessed the test-retest reliability of both approaches based on datasets of the same subjects acquired on two separate days. The novelty presented in this work is the feasibility to 
reliably identify networks in CBF data using either ICA or SBA and to quantify network activity i.e. CBF within the identified networks.

\section{Methods}

\section{Subjects}

Twelve healthy young right-handed subjects ( $5 \mathrm{~m} / 7 \mathrm{f}$; mean age \pm SD $23.9 \pm 2.3$ years; age range $19-27$ years) participated in this study and gave their written informed consent. Handedness was assessed with the Edinburg Handedness Scale (Oldfield 1971). Participants refrained from alcohol, caffeine and nicotine for at least $6 \mathrm{~h}$ prior to measurement and were not under any medication, did not consume drugs or had a history of psychiatric or neurologic disease. The study was approved by the ethical committee of Bern, Switzerland and was in agreement with the latest Declaration of Helsinki. The same subject group was previously analyzed and published in the study of Orosz et al. (2012).

\section{Data Acquisition}

All MRI scans were performed on a 3T Siemens Magnetom Trio (Erlangen, Germany) equipped with a 12-channel radio frequency head coil.

For the measurement of $\mathrm{CBF}$ a pseudo continuous arterial spin labeling (pCASL) technique with the following parameters was used (Dai et al. 2008; Wu et al. 2007): $\mathrm{TR} / \mathrm{TE}=3,000 / 18 \mathrm{~ms}$, field of view $(\mathrm{FOV})=220 \mathrm{~mm}^{2}$, matrix size $=64 \times 64$ (Isocenter of the readout slice was $90 \mathrm{~mm}$ above labeling plane), balanced labeling with mean Gz of $0.6 \mathrm{mT} / \mathrm{m}$ and 60 Hanning window-shaped RF pulses (RF duration $600 \mu$ s with $900 \mu$ s gap, a flip angle (FA) $=$ $25^{\circ}$, and bandwith $3,004 \mathrm{~Hz} /$ pixel), total labeling duration $(\tau)=1.72 \mathrm{~s}$ and post-labeling delay $(\mathrm{PLD})=1 \mathrm{~s}$. Five axial slices (EPI readout, $3.4 \mathrm{~mm}$ in-plane resolution, $10 \mathrm{~mm}$ slice thickness and $2.5 \mathrm{~mm}$ gap) were positioned parallel to the bi-commissural axis, and were shifted in z-direction to cover the SMN and the areas constituting the DMN. A total of 200 images, i.e. 100 pairs of label and control images were recorded in $10 \mathrm{~min}$. The sequence has been recorded with 3D PACE (Siemens Erlangen, Germany) to enable prospective motion correction.

After the pCASL run, a high resolution 3D T1-weighted, magnetization-prepared rapid-acquisition gradient echo (MP-RAGE) sequence was acquired (TR/TE 11.4/4.4 ms and $\mathrm{FA}=15^{\circ}, 176$ sagittal slices, thickness $=1.0 \mathrm{~mm}$, $\mathrm{FOV}=256 \mathrm{~mm} \times 256 \mathrm{~mm}$, matrix size $=256 \times 256$. The anatomical scan lasted approximately $4.5 \mathrm{~min}$.
TASK Design

During the pCASL runs subjects performed a simple blockdesign task with two conditions, rest and fingertapping. Each block lasted $30 \mathrm{~s}$ and each condition was repeated 10 times. A red cross in the middle of a black screen displayed via MR-compatible LCD goggles (VisuaStim XGA, Resonance technology Inc., Los Angeles, CA, USA) indicated a rest period, while a green cross signaled a fingertapping block. During fingertapping subjects sequentially opposed the thumb to index, medium, ring and little fingers of the non-dominant hand (i.e. the left hand) as quickly as possible. The sessions always started with a resting state condition block.

Each subject was recorded on two separate days at least 1 week apart (27.4 \pm 19.2 (range 7-63) days). The two recording sessions of each subject were randomly assigned to two different datasets (Data1/Data2) (Orosz et al. 2012).

\section{Data Analysis}

Preprocessing and analysis of MR data were performed in BrainVoyager QX (version 2.2, BrainInnovation, Maastricht, The Netherlands) and with own written MatLab (MathWorks, Natick, Massachusetts, USA) programs, statistical analyses (ANOVA) were computed in STATISTICA (StatSoftTM, version 10).

\section{CBF Quantification}

Raw pCASL images were motion-corrected using Levenberg-Marquarts's least square fit for six spatial parameters, coregistered to the individual anatomical scans (here the images were resampled into $3 \mathrm{~mm}$ isotropic voxels), rotated into the anterior-posterior commissural plane and normalized into standard Talairach space (Talairach and Tournoux 1988). Finally, the images were spatially smoothed with a Gaussian kernel (FWHM $10 \mathrm{~mm}$ ) to reduce interindividual anatomical differences and further increase the SNR (Wang et al. 2005). Quantification of CBF flow time series was based on the equation:

$C B F=\left(\frac{\lambda \cdot \Delta M}{2 \cdot \alpha \cdot M_{0} \cdot T_{1 b}}\right) \cdot\left(\frac{1}{e^{-w / T_{1 b}}-e^{\left.-(\tau+w) / T_{1 b}\right)}}\right)$

Post-labelling delay $(\omega)=1,000 \mathrm{~ms}$ (adjusted for each slice and the resampling factor), tagging duration $\tau=$ $1,720 \mathrm{~ms}$, blood/tissue water partition coefficient $\lambda=0.9 \mathrm{~g} / \mathrm{ml}$ and tagging efficiency assumed to be $\alpha=0.85$ (Wu et al. 2007). For 3.0T the decay time for labelled blood $\mathrm{T} 1 \mathrm{~b}=1,490 \mathrm{~ms}$, M0 are the equilibrium brain tissue magnetization images (Federspiel et al. 2006; Jann et al. 2010a; Wang et al. 2003c). $\Delta M$ was calculated by sinc-subtraction of label and control images. 
Sinc-subtraction has been demonstrated to efficiently minimize spurious BOLD contaminations within the CBF signal and thus was proposed as the method of choice for block-design experiments (Liu and Wong 2005; Wang et al. 2003a). Furthermore, Liu and Wong (2005) demonstrated that sinc-subtraction acts like a filter and is equivalent to other filtering approaches (Chuang et al. 2008; Liu and Wong 2005). Quantified CBF flow time series and also average CBF maps for the entire recording were calculated (Federspiel et al. 2006; Jann et al. 2010a).

\section{Seed Based Functional Connectivity Analysis (SBA)}

Seed regions were chosen based on the activation clusters published in (Orosz et al. 2012) determined by standard random effects GLM analyses to these datasets. For Data1 the center of gravity $\pm \mathrm{SD}$ (Talairch $\mathrm{x} / \mathrm{y} / \mathrm{z}$ ) and volume of the seed was $28.0 \pm 4.2 /-26.9 \pm 2.5 / 45.7 \pm 3.9 / 1,177$ $\mathrm{mm}^{3}$ and for Data2 it was $29.6 \pm 2.9 /-29.4 \pm 2.6 /$ $43.9 \pm 3.3 / 819 \mathrm{~mm}^{3}$. From these seeds the subjects CBF fluctuations were extracted and used as regressors in GLM analyses to define the subject specific functional connectivity maps. Group analysis was then performed using twosided $t$ test against zero to display the networks for either dataset. To probe for test-retest reliability we performed a $t$ test between the two datasets. T-maps of either dataset were thresholded with $p<0.05$ "Bonferroni" correction, while the group comparison t-map was corrected with a minimal cluster size threshold (0.05) estimated with Monte-Carlo simulations.

\section{ICA Decomposition and Identification of Networks}

To identify the FCNs on a pure data driven basis, we used a temporally concatenated group level ICA approach (Calhoun et al. 2001, 2004; Horn et al. 2012). For this purpose, the individual flow time series were normalized (scaled between 0 and 1) to reduce the inter-subject variability in the CBF data while maintaining the relative temporal signal fluctuations. The concatenated dataset was then subjected to the ICA algorithm (FastICA developed by Hyvarinen \& Oja (Hyvarinen and Oja 2000) implemented in BrainVoyager QX) and decomposed into 30 components. Concatenating the individual datasets has the advantage that only one set of ICs is generated. Thus, no second level grouping of individual ICs has to be performed in contrast to approaches performing the ICA decomposition on the subject level (Calhoun et al. 2001; Esposito et al. 2005; Jann et al. 2009, 2010b). The stimulation design was used to create a box-car predictor (convolved with a double gamma hemodynamic response function) to identify the network that best represented the temporal signature of this predictor. Each of the estimated group components (GC) had a correlation coefficient that indicated the similarity of its temporal fluctuations with the stimulation predictor, and the one displaying the highest temporal correlation were selected. Average network timeserie of this component across subjects was calculated based on the GC timeserie subdivided into periods corresponding to the separate subjects and rescaled from 0 to 1 for each subject.

\section{Estimation of Subject Specific Maps (SMs)}

Based on the group ICA results we calculated for each subject its specific spatial map using a back-reconstruction approach based on dual spatio-temporal regression (Calhoun et al. 2004; Horn et al. 2012). Therefore we used the z-transformed GC timeseries (segmented into the timeperiods corresponding to the individual subjects) as regressors in single subject GLMs (dependent variable were the original, unscaled CBF timeseries; motion parameters were not included in the GLM (Erhardt et al. 2010). This yielded the spatial pattern of voxels that correlate with the timeseries of the GC in the specific single subject. To cross validate the single subject GLM results with the GC and to estimate the test-retest robustness of the approach, we performed a $t$ test against zero across the SMs on Data1 and Data2.

ASL, in addition, provides the possibility to quantify the total absolute CBF within the network (either group or individual networks). Hence, the quantification of intersubject differences that we refer to in the manuscript is the subject specific quantified CBF within the group network (see next "Quantification of network CBF" subsection).

\section{Quantification of Network CBF}

For the quantification of network $\mathrm{CBF}$ we defined regions of interest (ROI) based on the thresholded GC z-maps. For the SMN we considered only areas showing a positive correlation with the task predictor, i.e. a positive polarity of the GC. For the DMN we selected the clusters with negative polarity. For these ROIs the original, unscaled individual CBF time series were extracted. As described above in "ICA decomposition and identification of networks" subsection for the mean GC timeseries, we also computed the temporal fluctuations of the CBF signal in the two FCNs for each individual subjects. These CBF time series then were averaged across subjects and the correlations between the two FCNs and the task predictor were computed.

Based on the defined ROIs, the mean network CBF during rest and fingertapping conditions were calculated. To this end, individual perfusion time series were separated into the blocks associated to either task condition, averaged 
first within subject and then across subjects. A $2 \times 2 \times 2$ repeated measures Analysis of Variance (ANOVA) with the within-subject factors networks (DMN/SMN), condition (rest/fingertapping) and dataset (Data1/Data2) was performed to test for significant network and condition effects and test-retest reliability. Significant interactions were further analyzed with the Tukey's Honestly Significant Difference (HSD) post hoc test.

\section{Estimation of Signal-to-Noise Ratio (SNR)}

The SNR of individual CBF datasets was estimated as follows. First, for each subject the global CBF signal averaged across all grey matter (GM) voxels was extracted. Second, the individual SNR was defined as the ratio of the temporal mean of the GM and its standard deviation (Donahue et al. 2009). Finally the mean SNR and its standard deviation were calculated across subjects. Similarly, the SNRs for the periods corresponding to the rest and fingertapping conditions were calculated respectively. A two-sided paired-sample $t$ test was applied for comparing $\mathrm{SNR}_{\text {data1 }}$ and $\mathrm{SNR}_{\text {data2 }}$ as well as for comparing $\mathrm{SNR}_{\text {task }}$ and $\mathrm{SNR}_{\text {rest }}$.

The same analysis was performed for the scaled $\mathrm{CBF}$ time series that were submitted to the ICA to estimate the effect of the scaling on SNR.

\section{Results}

The group SBA approach was able to identify the SMN (task specific positive FCN) in the time series of the ASL datasets (Fig. 1), whereas the concatenated group ICA approach, not only revealed the SMN, but also the DMN (task negative FCN) (Fig. 2/Table 1). Mean CBF values of and relative changes between rest and fingertapping conditions for both networks as identified by ICA are listed in Table 2 and depicted in Fig. 3.

\section{Correlation Results}

The SMN was positively correlated with the task predictor ( $r=0.31 p<0.002)$ whereas the DMN was negatively correlated to it $(r=-0.27 p<0.007)$.

The mean global CBF values of Data1 and Data2 showed a highly significant correlation $(r=0.82 p<0.0006 /$ Fig. 4).

\section{Subject Specific Map Results}

The Z-maps of the $t$ tests across the ICA SMs of either dataset revealed spatial patterns similar to the ICA GC. Moreover, the paired $t$ test between Data1 and Data2 did not show any significant difference at $\mathrm{p}<0.01$ with a cluster size threshold of 10 voxels (Fig. 5) indicating testretest reliability. The maps calculated based on the SBA approach displayed a similar pattern as the SMN of the ICA result. However, the SMN comparison for Data1 and Data2 in the SBA showed significant differences especially in the right motor areas.

ANOVA Results of FCN Quantification

The repeated measures ANOVA showed no statistically significant effect of dataset $(F(1,11)=4.7 p>0.05)$ and no significant interactions involving the factor dataset. Significant effects were found for condition $(F(1,11)=$ $21.8, p<0.001)$ and the interaction of network*condition $(F(1,11)=86.5, p<0.00001)$. The post hoc test revealed that the significant interaction arose from significantly higher $\mathrm{CBF}$ in the SMN compared to DMN during fingertapping ( $p<0.0002)$. Moreover, $\mathrm{CBF}$ was significantly higher in the SMN during fingertapping than during rest $(p<0.0002)$, while in the DMN no such effect was observed.

\section{SNR}

The average SNR of the CBF images over both datasets and across the whole recording was $3.15 \pm 1.62$ and the $t$ test for SNRs between Data1 and Data2 revealed no statistical difference $(t(11)=-0.48, p=0.64)$. The estimated SNR for the separate conditions were $\mathrm{SNR}_{\text {rest }}=$ $3.43 \pm 1.88$ and $\mathrm{SNR}_{\text {task }}=4.01 \pm 1.85$. Again, the $t$ test for $\mathrm{SNR}_{\text {task }} \mathrm{vs} \mathrm{SNR}_{\text {rest }}(t=1.08, p=0.29)$ and the $t$ test for $\mathrm{SNR}_{\text {rest_data1 }}$ vs $\operatorname{SNR}_{\text {rest_data2 }}(t=-0.46, p=0.65)$ were not significant.

The SNR estimations of the scaled CBF time series that were used for ICA were as follows: $6.90 \pm 1.47$ for joint Data1 and Data2 (no statistical significance between Data1 and Data2: $t=-0.58, p=0.57), \mathrm{SNR}_{\text {rest }}=7.41 \pm 2.20$ and $\mathrm{SNR}_{\text {task }}=8.71 \pm 2.39$ (again no statistical difference between datasets: $t=-1.09 \mathrm{p}=0.29$ for rest and $t=1.28$, $p=0.21$ for task respectively).

\section{Discussion}

The main aim of this study was to explore the capability of ICA and SBA to identify FCN in ASL data, which then would allow to absolutely quantifying network CBF. This would overcome one of the main limitations of the networks identified in fMRI BOLD data, which provide relative measures of network activity. Our study is one of the first that investigated the feasibility of ICA based network analysis in ASL data (Liang et al. 2011; Xie et al. 2011) in 


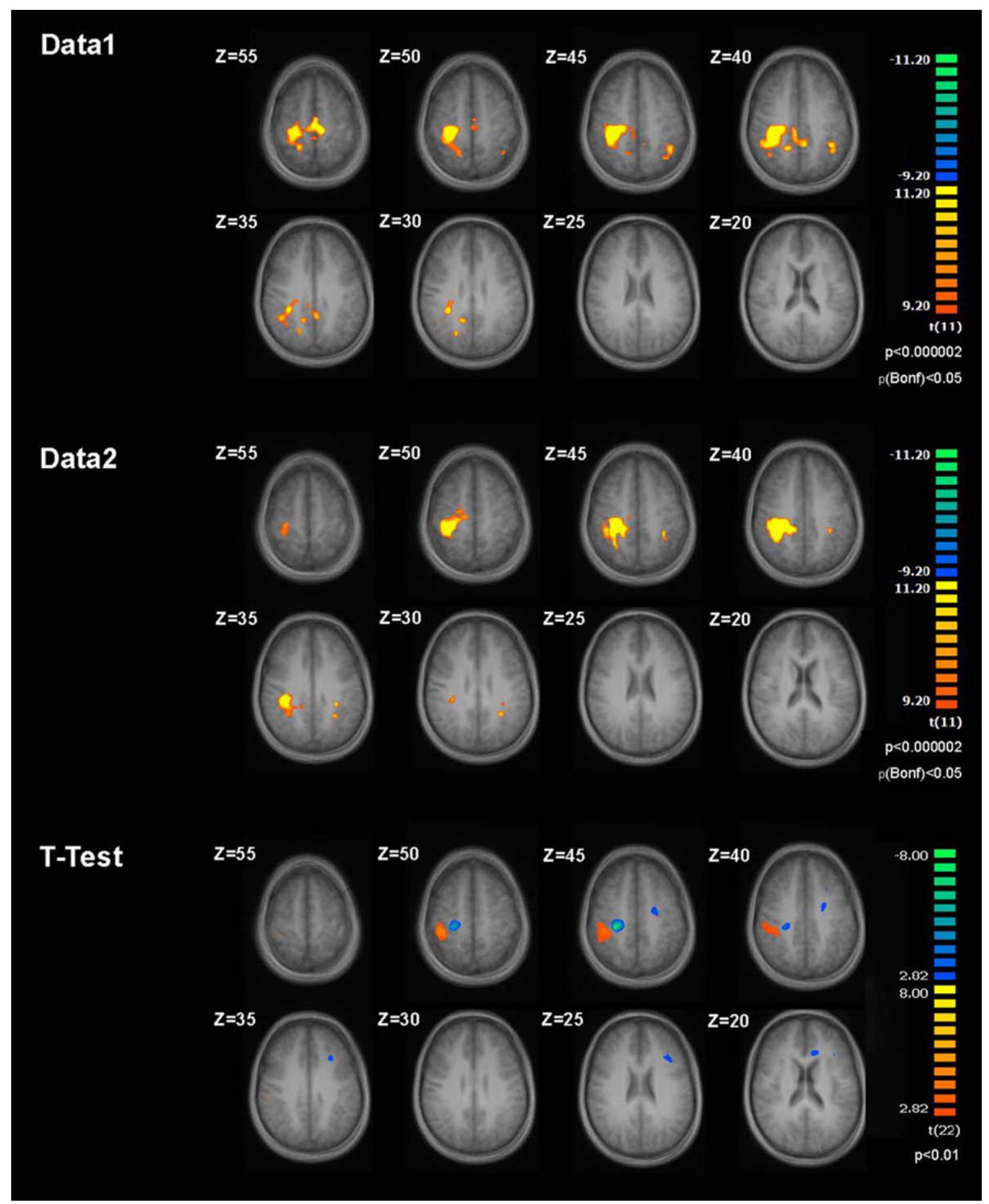

Fig. $1 t$ test across all 12 subjects' SMs based on SBA for Data1 and Data2, respectively. The $t$ test comparing the individual maps of Data1 versus Data2 displays statistically significant differences in the areas corresponding to different seeds in the motor cortex

comparison to the SBA, and demonstrated the possibility to analyze networks without the a-priory selection of a seed region (Chuang et al. 2008; Zou et al. 2009). The presented approach of concatenating individual ASL datasets indeed proved capable of identifying the network related to the execution of the motor task (Somato-Motor Network; SMN) as well as the (nodes of the) Default Mode Network
(DMN). Both networks comprise the brain regions that have been consistently reported in FMRI BOLD literature (Damoiseaux et al. 2006; De Luca et al. 2006; Fox et al. 2005; Jann et al. 2010b; Lowe et al. 1998). The SMs representing the subject specific spatial pattern of the network were consistent with the GC as well as robust for Data1 and Data2. The temporal fluctuations of the identified networks 


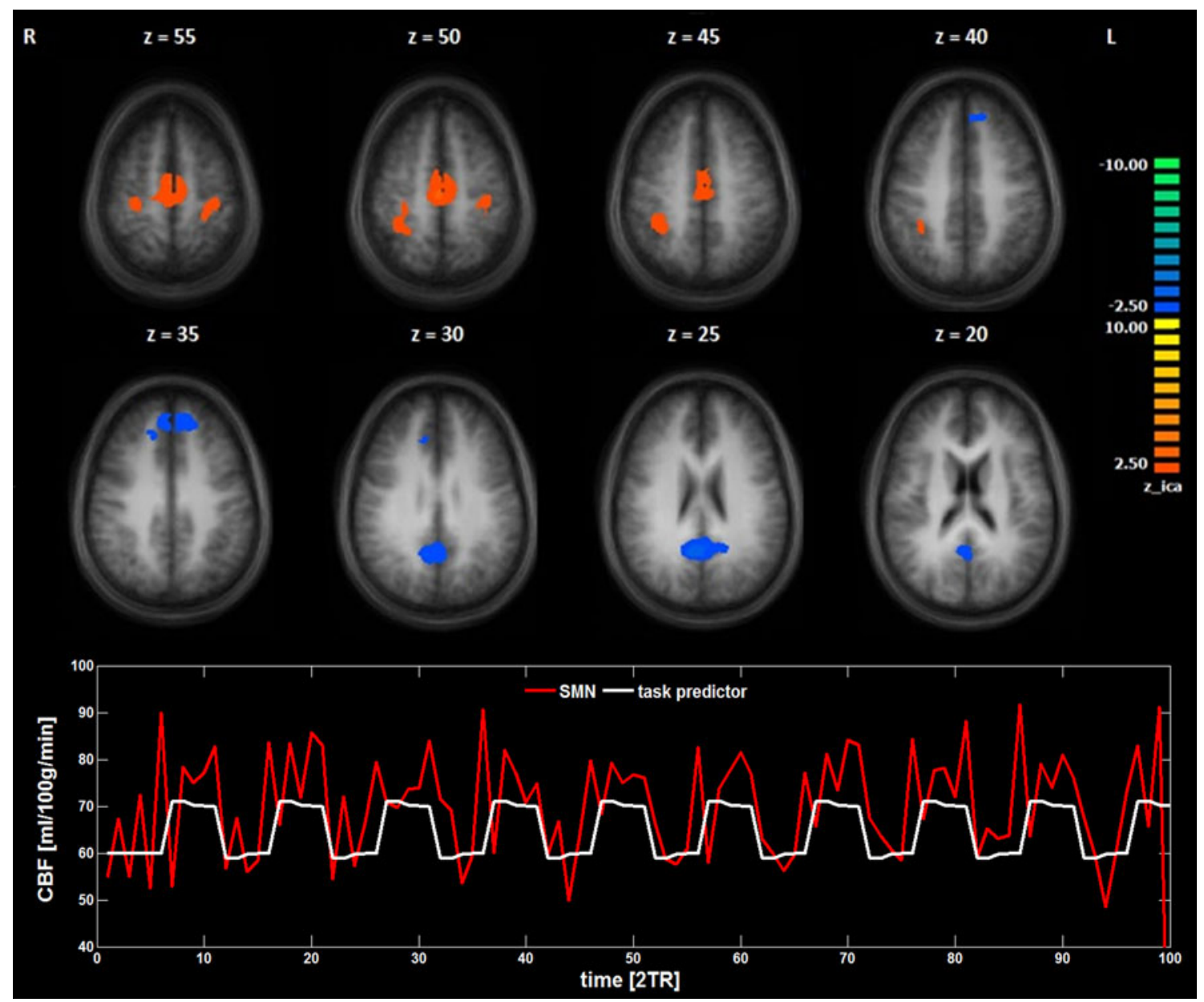

Fig. 2 The upper part shows the axial slices of the IC factor representing the SMN and DMN networks. The lower plot depicts the temporal dynamics of the SMN (red line) and the task predictor (white line) (Color figure online)

Table 1 Regions of interest constituting the two networks. (Coordinates are in Talairach space.)

\begin{tabular}{lccccc}
\hline & \multicolumn{2}{l}{ Center of gravity \pm SD } & & No. of voxels \\
\cline { 2 - 4 } & $\mathrm{x}$ & $\mathrm{y}$ & $\mathrm{z}$ & \\
\hline SMN & $4.5 \pm 19.6$ & $-21.0 \pm 11.7$ & $50.8 \pm 4.1$ & 8,958 & BA \\
Motor right & $31.4 \pm 3.8$ & $-36.0 \pm 7.7$ & $48.6 \pm 4.5$ & 2,317 & $3 / 4$ \\
Motor left & $-30.3 \pm 4.0$ & $-25.9 \pm 4.4$ & $52.9 \pm 2.7$ & 1,183 & Postcentral gyrus \\
SMA & $0.6 \pm 5.7$ & $-13.6 \pm 6.5$ & $51.3 \pm 3.8$ & 5,458 & Medial fronal gyrus \\
DMN & $0.1 \pm 8.6$ & $-17.7 \pm 45.6$ & $29.3 \pm 5.3$ & 6,294 & 6 \\
Frontal right & $8.3 \pm 4.8$ & $40.6 \pm 5.4$ & $34.2 \pm 1.7$ & 878 & Medial fronal gyrus \\
Frontal left & $-10.1 \pm 4.6$ & $43.2 \pm 3.5$ & $35.5 \pm 2.4$ & 1,417 & Medial fronal gyrus \\
PCC & $1.8 \pm 7.0$ & $-52.1 \pm 4.2$ & $26.0 \pm 3.5$ & 3,999 & 9 \\
\hline
\end{tabular}

were positively (SMN) and negatively (DMN) correlated with the task predictor. Hence, both networks are in good agreement with BOLD ICA networks in terms of their spatial pattern and their temporal dynamics.

In contrast, the SBA only identified the SMN while the DMN could not be detected. This might be due to the scaling and concatenation of the datasets before the ICA or due to some necessary preprocessing steps for ICA such as pre-whitening and dimensionality reduction that is not required for SBA (Joel et al. 2011). Nevertheless, the SMs were computed based on the same, unscaled perfusion timeseries using a GLM. Only the regressors used for a 
Table 2 Mean CBF values for network, dataset and condition (cf. Fig. 3) and relative CBF changes between rest and fingertapping task (FT)

\begin{tabular}{llllll}
\hline & \multicolumn{1}{l}{ DMN } & & \multicolumn{2}{l}{ SMN } \\
\cline { 2 - 3 } \cline { 5 - 6 } & Data1 & Data2 & Data1 & $74.60 \pm 8.57$ \\
\hline FT $(\mathrm{ml} / 100 \mathrm{~g} / \mathrm{min})$ & $68.98 \pm 4.41$ & $62.10 \pm 4.41$ & & $76.93 \pm 10.37$ & $62.86 \pm 6.90$ \\
REST $(\mathrm{ml} / 100 \mathrm{~g} / \mathrm{min})$ & $67.26 \pm 8.03$ & $64.94 \pm 6.90$ & & $63.35 \pm 5.81$ & $0.15 \pm 0.09$ \\
\% change & $0.03 \pm 0.08$ & $-0.05 \pm 0.07$ & & $0.17 \pm 0.08$ & \\
\hline
\end{tabular}
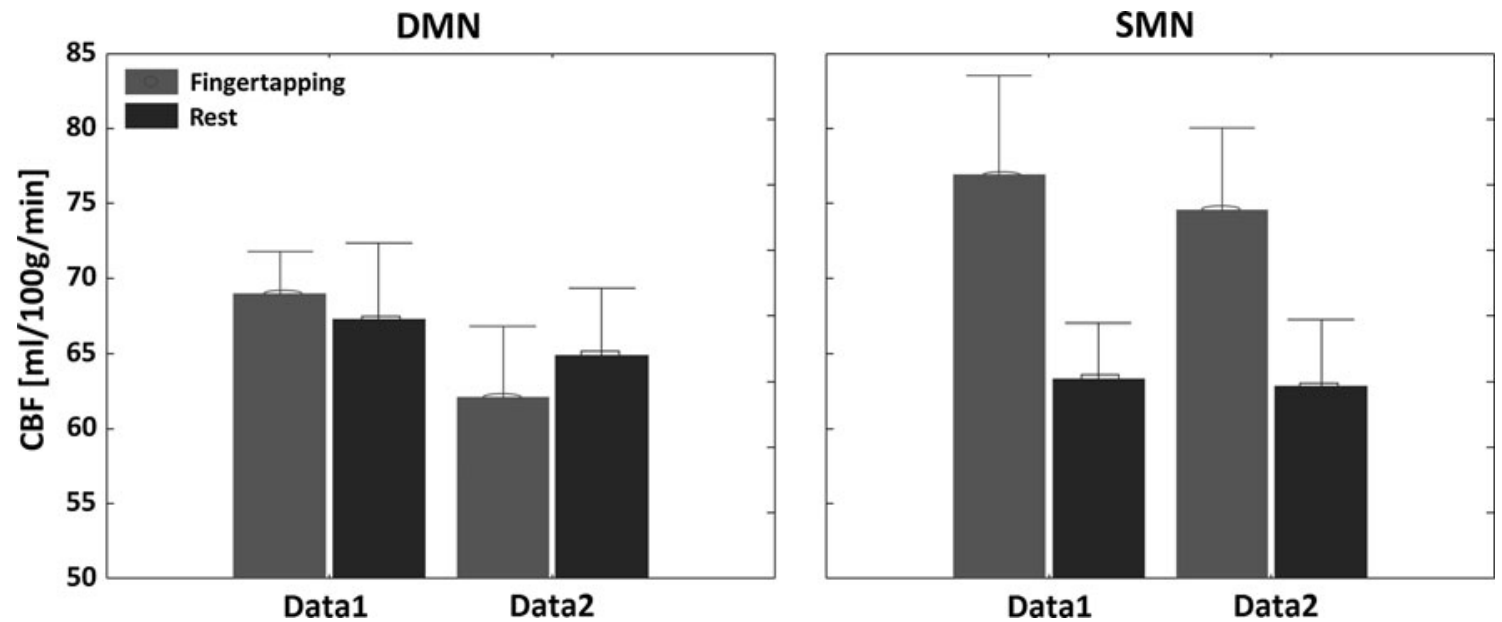

Fig. 3 Mean CBF values for network, dataset and condition

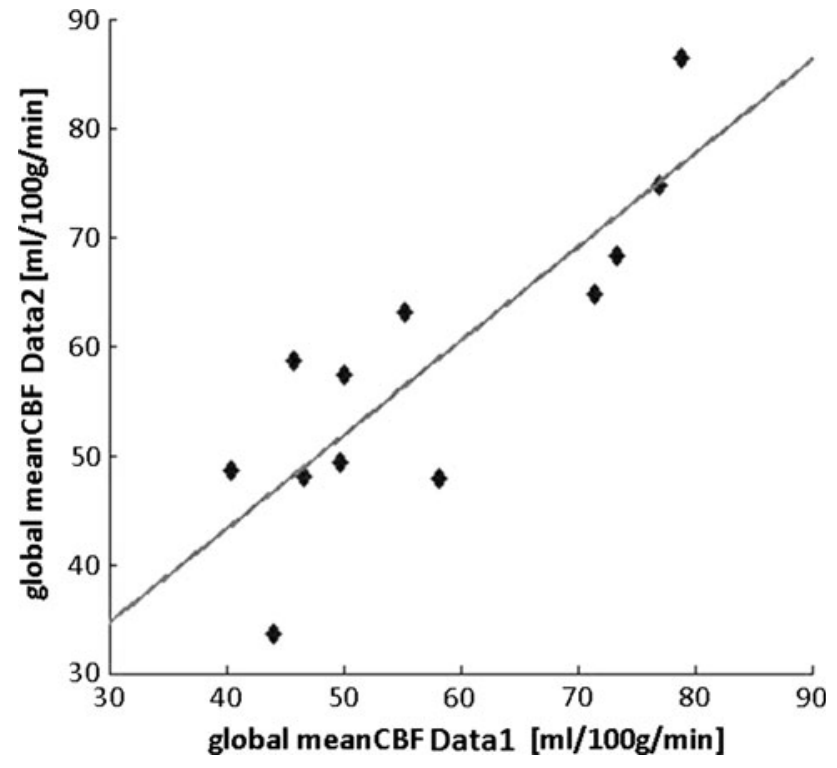

Fig. 4 Global mean CBF of all participants compared between Data1 and Data2. Correlation between Data1 and Data2 was $r=0.82$, $p=0.0006, R^{2}=0.68$

specific subject differed between SMs for ICA and SBA respectively. In the case of ICA, it was the global network dynamics, whereas for the SBA the temporal fluctuations of the CBF signal in the seed (motor cortex) were used. Furthermore, we found significant differences of the SBA deduced networks for Data1 and Data2, specifically in the motor cortex. This finding can be explained when considering the seeds used for either dataset. The seeds were chosen based on a random effects GLM using the fingertapping task-predictor as the regressor (Orosz et al. 2012). The significant differences were exactly in the spots where the two seeds differed. This finding of slightly deviant networks resulting from different seeds is well in line and confirms previous studies (Buckner et al. 2008; Cole et al. 2010). Thus, SBA might be the method of choice when clear hypotheses exist and very specific functional connectivity networks are aimed to be investigated. However, the seed region bias should always be kept in mind. ICA on the other side is a data driven approach that is suitable to fully explore the functional connectivity of a dataset (Joel et al. 2011).

Moreover, in the present study we also attempted to quantify network activity by means of absolute CBF. The absolute CBF values extracted for both networks and during rest or task condition, as well as the percentage increase were in agreement with values reported in literature ((Wang et al. 2003b) ASL/(Colebatch et al. 1991) PET). The ANOVA results of the CBF values further demonstrated the stability and thus test-retest reliability of the presented approach. In fact, there was no statistically significant difference between Data1 and Data2. However, the DMN in Datal showed a non-significant higher mean 


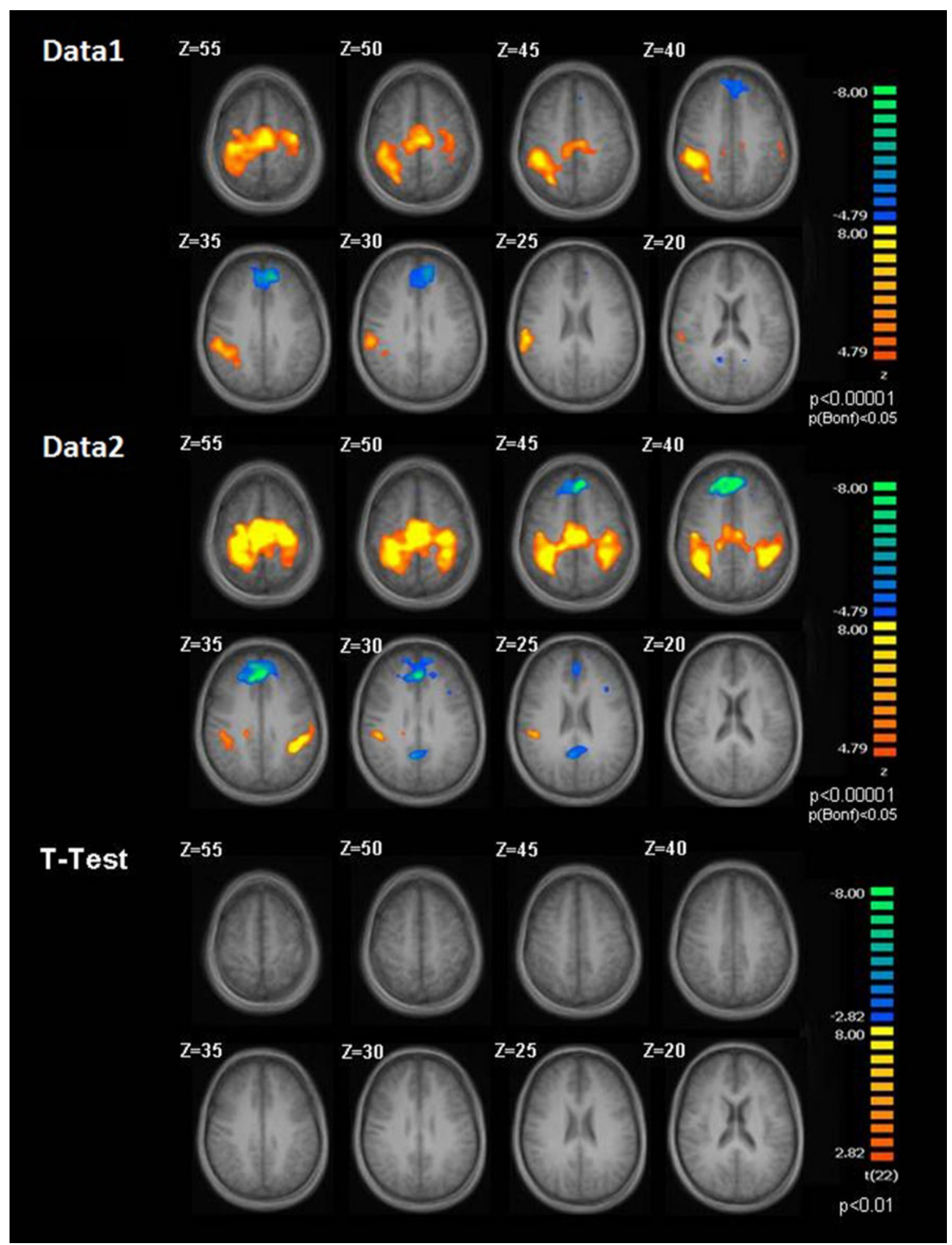

Fig. 5 Z-maps (based on a $t$ test across all 12 subjects' SMs based on ICA) for Data1 and Data2, respectively. The $t$ test comparing the individual maps of Data1 versus Data2 displays no statistically significant difference

CBF during task than during rest. Possible explanations might on the one hand be related to the SNR during resting state and on the other hand to the low cognitive demand of the task. While on the group data the separation of task and rest and the SNR of the signal are sufficient to reliably identify the networks, whereas on the single subject level the low SNR during rest might confound the analysis. An effect that has previously been observed by Biswal et al. 
(1997) who reported on higher standard deviation in perfusion data during rest than task and was reproduced in this study. This, however, does not apply to the quantification of the CBF values themselves but only to the differentiation of networks during rest. A more plausible explanation for the inability to find a decrease of DMN CBF during task execution on Datal might be given by the insufficient modulation of activity in the DMN due to very low cognitive demand. It is known that the DMN deactivates during the task and this deactivation increases with task difficulty (Esposito et al. 2006; Raichle and Snyder 2007). The assumption of relative low modulation of DMN activity by the fingertapping task is supported by the high standard deviation in the DMN decrease as compared to the SMN. This might also explain why at the chosen statistical threshold for the region of interest analysis, the DMN did not show the areas in the inferior parietal lobes. At a slightly lower threshold for the network GC (i.e. $z=2$ ) these areas were present.

This observation will have implications for future studies and for the application of the approach to pure resting state ASL data. Usually ASL scans acquire fewer averages and whether the SNR of the quantified CBF fluctuations might be sufficient to reliably identify networks also during rest needs to be demonstrated. Besides these theoretical considerations about possible problems hampering the application of this approach to resting state measurements, there is some empirical evidence suggesting the practicability: a few studies, using SBA approaches though, demonstrated the feasibility to detect FCN in ASL data (Biswal et al. 1997; Chuang et al. 2008; Zou et al. 2009). In addition, SNR can be increased by longer ASL recordings (more volumes) or using large sample sizes (more subjects).

One limitation of the present study was the incomplete coverage of brain that was restricted to the dorsal/superior part, due to the tradeoff between coverage and temporal resolution since increasing the coverage would cost longer TRs. Since for perfusion time series the temporal resolution is halved with respect to the resolution of the sequence (pairwise acquisition of label and control images) it is an issue for future studies but eventually could be overcome by novel faster ASL sequences (Fernandez-Seara et al. 2008).

Another caveat are possible phase-tracking errors in pCASL (Wu et al. 2007) due to field inhomogeneity effects at the labeling location that can affect labeling efficiency $(\alpha)$ and consequentially underestimate CBF (Jung et al. 2010). Although $\alpha$ in pCASL can be $\geq 80 \%$ at frequency offset around 1 ppm (Dai et al. 2008), it still might vary within subjects and accordingly increase test-retest variability (Wu et al. 2011). Recent advantages in pCASL acquisition and post-processing strategies could however help to reduce inter-session and -subject variability (Jahanian et al. 2011; Jung et al. 2010).
In summary, the novel approach of ICA based ASL analysis reported here provides a new strategy to evaluate differences of networks besides the comparison of subject specific maps (SM). ASL, in addition to BOLD, allows assessing the level of network activity in terms of quantified CBF within the network, which is completely inaccessible for BOLD. Thus ASL network analysis allows quantifying inter-individual differences in the resting state and task-related activations and deactivations.

Moreover, the presented approach might be of particular interest in investigation of dys-functions in psychiatric and neurologic disorders, e.g. catatonia, schizophrenia, depression or Alzheimer's disease. Specifically a quantitative analysis of absolute network CBF and their relation to pathophysiological rating scales could help to better understand the baseline alterations in patient groups and even their inter-individual differences associated to cognitive and behavioral impairments.

\section{References}

Beckmann CF, DeLuca M, Devlin JT, Smith SM (2005) Investigations into resting-state connectivity using independent component analysis. Philos Trans R Soc Lond B 360(1457):1001-1013

Biswal B, Yetkin FZ, Haughton VM, Hyde JS (1995) Functional connectivity in the motor cortex of resting human brain using echo-planar MRI. Magn Reson Med 34(4):537-541

Biswal BB, Van Kylen J, Hyde JS (1997) Simultaneous assessment of flow and BOLD signals in resting-state functional connectivity maps. NMR Biomed 10(4-5):165-170

Bokkers RP, van Laar PJ, van de Ven KC, Kapelle LJ, Klijn CJ, Hendrikse J (2008) Arterial spin-labeling MR imaging measurements of timing parameters in patients with a carotid artery occlusion. AJNR Am J Neuroradiol 29(9):1698-1703

Bokkers RP, Bremmer JP, van Berckel BN, Lammertsma AA, Hendrikse J, Pluim JP, Kappelle LJ, Boellaard R, Klijn CJ (2010) Arterial spin labeling perfusion MRI at multiple delay times: a correlative study with $\mathrm{H}(2)(15) \mathrm{O}$ positron emission tomography in patients with symptomatic carotid artery occlusion. J Cereb Blood Flow Metab 30(1):222-229

Broyd SJ, Demanuele C, Debener S, Helps SK, James CJ, SonugaBarke EJ (2009) Default-mode brain dysfunction in mental disorders: a systematic review. Neurosci Biobehav Rev 33(3): 279-296

Buckner RL, Andrews-Hanna JR, Schacter DL (2008) The brain's default network: anatomy, function, and relevance to disease. Ann N Y Acad Sci 1124:1-38

Buxton RB, Uludag K, Dubowitz DJ, Liu TT (2004) Modeling the hemodynamic response to brain activation. Neuroimage 23(Suppl 1):S220-S233

Calhoun VD, Adali T, Pearlson GD, Pekar JJ (2001) A method for making group inferences from functional MRI data using independent component analysis. Hum Brain Mapp 14(3): 140-151

Calhoun VD, Adali T, Pekar JJ (2004) A method for comparing group fMRI data using independent component analysis: application to visual, motor and visuomotor tasks. Magn Reson Imaging 22(9):1181-1191 
Chuang KH, van Gelderen P, Merkle H, Bodurka J, Ikonomidou VN, Koretsky AP, Duyn JH, Talagala SL (2008) Mapping restingstate functional connectivity using perfusion MRI. Neuroimage 40(4):1595-1605

Cole DM, Smith SM, Beckmann CF (2010) Advances and pitfalls in the analysis and interpretation of resting-state FMRI data. Front Syst Neurosci 4:8

Colebatch JG, Deiber MP, Passingham RE, Friston KJ, Frackowiak RS (1991) Regional cerebral blood flow during voluntary arm and hand movements in human subjects. J Neurophysiol 65(6): 1392-1401

Dai W, Garcia D, de Bazelaire C, Alsop DC (2008) Continuous flowdriven inversion for arterial spin labeling using pulsed radio frequency and gradient fields. Magn Reson Med 60(6): 1488-1497

Dai W, Varma G, Scheidegger R, Shankaranarayanan A, Schlaug G, Alsop D (2012) Resting fluctuations in volumetric arterial spin labeling. In: Proceedings of the international society of magnetic resonance in medicine, Melbourne, 2012

Damoiseaux JS, Rombouts SA, Barkhof F, Scheltens P, Stam CJ, Smith SM, Beckmann CF (2006) Consistent resting-state networks across healthy subjects. Proc Natl Acad Sci USA 103(37): 13848-13853

De Luca M, Beckmann CF, De Stefano N, Matthews PM, Smith SM (2006) fMRI resting state networks define distinct modes of longdistance interactions in the human brain. Neuroimage 29(4): 1359-1367

Detre JA, Leigh JS, Williams DS, Koretsky AP (1992) Perfusion imaging. Magn Reson Med 23(1):37-45

Donahue MJ, Blicher JU, Ostergaard L, Feinberg DA, MacIntosh BJ, Miller KL, Gunther M, Jezzard P (2009) Cerebral blood flow, blood volume, and oxygen metabolism dynamics in human visual and motor cortex as measured by whole-brain multimodal magnetic resonance imaging. J Cereb Blood Flow Metab 29(11):1856-1866

Duong TQ, Kim DS, Ugurbil K, Kim SG (2001) Localized cerebral blood flow response at submillimeter columnar resolution. Proc Natl Acad Sci USA 98(19):10904-10909

Erhardt EB, Rachakonda S, Bedrick EJ, Allen EA, Adali T, Calhoun VD (2010) Comparison of multi-subject ICA methods for analysis of fMRI data. Hum Brain Mapp 32(12):2075-2095

Esposito F, Scarabino T, Hyvarinen A, Himberg J, Formisano E, Comani S, Tedeschi G, Goebel R, Seifritz E, Di Salle F (2005) Independent component analysis of fMRI group studies by selforganizing clustering. Neuroimage 25(1):193-205

Esposito F, Bertolino A, Scarabino T, Latorre V, Blasi G, Popolizio T, Tedeschi G, Cirillo S, Goebel R, Di Salle F (2006) Independent component model of the default-mode brain function: assessing the impact of active thinking. Brain Res Bull 70(4-6):263-269

Federspiel A, Muller TJ, Horn H, Kiefer C, Strik WK (2006) Comparison of spatial and temporal pattern for fMRI obtained with BOLD and arterial spin labeling. J Neural Transm 113(10): $1403-1415$

Fernandez-Seara MA, Edlow BL, Hoang A, Wang J, Feinberg DA, Detre JA (2008) Minimizing acquisition time of arterial spin labeling at 3T. Magn Reson Med 59(6):1467-1471

Fox MD, Snyder AZ, Vincent JL, Corbetta M, Van Essen DC, Raichle ME (2005) The human brain is intrinsically organized into dynamic, anticorrelated functional networks. Proc Natl Acad Sci USA 102(27):9673-9678

Greicius M (2008) Resting-state functional connectivity in neuropsychiatric disorders. Curr Opin Neurol 21(4):424-430

Horn H, Jann K, Federspiel A, Walther S, Wiest R, Müller T, Strik WK (2012) Semantic network disconnection in formal thought disorder. Neuropsychobiology 66:14-23
Hyvarinen A, Oja E (2000) Independent component analysis: algorithms and applications. Neural Netw 13(4-5):411-430

Jahanian H, Noll DC, Hernandez-Garcia L (2011) B0 field inhomogeneity considerations in pseudo-continuous arterial spin labeling (pCASL): effects on tagging efficiency and correction strategy. NMR Biomed 4(10):1202-1209

Jann K, Dierks T, Boesch C, Kottlow M, Strik W, Koenig T (2009) BOLD correlates of EEG alpha phase-locking and the fMRI default mode network. Neuroimage 45(3):903-916

Jann K, Koenig T, Dierks T, Boesch C, Federspiel A (2010a) Association of individual resting state EEG alpha frequency and cerebral blood flow. Neuroimage 51(1):365-372

Jann K, Kottlow M, Dierks T, Boesch C, Koenig T (2010b) Topographic electrophysiological signatures of FMRI resting state networks. PLoS ONE 5(9):e12945

Joel SE, Caffo BS, van Zijl PC, Pekar JJ (2011) On the relationship between seed-based and ICA-based measures of functional connectivity. Magn Reson Med 66(3):644-657

Jung Y, Wong EC, Liu TT (2010) Multiphase pseudocontinuous arterial spin labeling (MP-PCASL) for robust quantification of cerebral blood flow. Magn Reson Med 64(3):799-810

Liang X, Tournier J-D, Masterton R, Connelly A, Calamante F (2011) An improved 3D GRASE pCASL method for whole-brain restingstate functional connectivity. In: Proceedings of the international society of magnetic resonance in medicine, Montreal, 2011

Liang X, Tournier J-D, Masterton R, Connelly A, Calamante F (2012) A k-space sharing 3D GRASE pseudocontinuous ASL method for whole-brain resting-state functional connectivity. Int $\mathrm{J}$ Imaging Syst Technol 22:37-43

Liu TT, Wong EC (2005) A signal processing model for arterial spin labeling functional MRI. Neuroimage 24(1):207-215

Lowe MJ, Mock BJ, Sorenson JA (1998) Functional connectivity in single and multislice echoplanar imaging using resting-state fluctuations. Neuroimage 7(2):119-132

Luh WM, Wong EC, Bandettini PA, Hyde JS (1999) QUIPSS II with thin-slice TI1 periodic saturation: a method for improving accuracy of quantitative perfusion imaging using pulsed arterial spin labeling. Magn Reson Med 41(6):1246-1254

Oldfield RC (1971) The assessment and analysis of handedness: the Edinburgh inventory. Neuropsychologia 9(1):97-113

Orosz A, Jann K, Wirth M, Wiest R, Dierks T, Federspiel A (2012) Theta burst TMS increases cerebral blood flow in the primary motor cortex during motor performance as assessed by arterial spin labeling (ASL). Neuroimage 61(3):599-605

Raichle ME, Snyder AZ (2007) A default mode of brain function: a brief history of an evolving idea. Neuroimage 37(4):1083-1090 (discussion 1097-1089)

Raichle ME, MacLeod AM, Snyder AZ, Powers WJ, Gusnard DA, Shulman GL (2001) A default mode of brain function. Proc Natl Acad Sci USA 98(2):676-682

Talairach J, Tournoux P (eds) (1988) Co-planar stereotactic atlas of the human brain. Thieme, New York

Wang J, Aguirre GK, Kimberg DY, Detre JA (2003a) Empirical analyses of null-hypothesis perfusion FMRI data at 1.5 and $4 \mathrm{~T}$. Neuroimage 19(4):1449-1462

Wang J, Aguirre GK, Kimberg DY, Roc AC, Li L, Detre JA (2003b) Arterial spin labeling perfusion fMRI with very low task frequency. Magn Reson Med 49(5):796-802

Wang J, Alsop DC, Song HK, Maldjian JA, Tang K, Salvucci AE, Detre JA (2003c) Arterial transit time imaging with flow encoding arterial spin tagging (FEAST). Magn Reson Med 50(3):599-607

Wang J, Rao H, Wetmore GS, Furlan PM, Korczykowski M, Dinges DF, Detre JA (2005) Perfusion functional MRI reveals cerebral blood flow pattern under psychological stress. Proc Natl Acad Sci USA 102(49):17804-17809 
Williams DS, Detre JA, Leigh JS, Koretsky AP (1992) Magnetic resonance imaging of perfusion using spin inversion of arterial water. Proc Natl Acad Sci USA 89(1):212-216

Wu WC, Fernandez-Seara M, Detre JA, Wehrli FW, Wang J (2007) A theoretical and experimental investigation of the tagging efficiency of pseudocontinuous arterial spin labeling. Magn Reson Med 58(5):1020-1027

Wu CW, Gu H, Lu H, Stein EA, Chen JH, Yang Y (2009) Mapping functional connectivity based on synchronized CMRO2 fluctuations during the resting state. Neuroimage 45(3):694-701

Wu WC, Jiang SF, Yang SC, Lien SH (2011) Pseudocontinuous arterial spin labeling perfusion magnetic resonance imaging-a normative study of reproducibility in the human brain. Neuroimage 56(3):1244-1250
Xie J, Jezzard P, Okell T, Miller K, Chappell M, Smith S (2011) Resting state network study of quantitative perfusion fluctuations. In: Proceedings of the organization for human brain mapping, Quebec City, 2011

Ye FQ, Berman KF, Ellmore T, Esposito G, van Horn JD, Yang Y, Duyn J, Smith AM, Frank JA, Weinberger DR, McLaughlin AC (2000) $\mathrm{H}(2)(15) \mathrm{O}$ PET validation of steady-state arterial spin tagging cerebral blood flow measurements in humans. Magn Reson Med 44(3):450-456

Zou Q, Wu CW, Stein EA, Zang Y, Yang Y (2009) Static and dynamic characteristics of cerebral blood flow during the resting state. Neuroimage 48(3):515-524 